\title{
Quantum populations near black-hole singularities
}

\author{
Ludwig Eglseer $\odot,{ }^{1, *}$ Stefan Hofmann $\odot^{1, \dagger}$ and Marc Schneider $\circledast^{2, *}$ \\ ${ }^{1}$ Arnold Sommerfeld Center for Theoretical Physics, Theresienstraße 37, 80333 München, Germany \\ ${ }^{2}$ Albert-Einstein Institute for Gravitational Physics, Am Mühlenberg 1, 14476 Potsdam, Germany
}

(Received 23 December 2019; accepted 19 October 2021; published 11 November 2021)

\begin{abstract}
Schwarzschild black-hole interiors border on spacelike singularities representing classical information leaks. We show that local quantum physics is decoupled from these leaks due to dynamically generated boundaries, called Zeno borders. Beyond Zeno borders black-hole interiors become asymptotically silent, and quantum fields evolve freely toward the geodesic singularity with vanishing probability measure for populating the geodesic boundary. Thus Zeno borders represent a probabilistic completion of Schwarzschild black holes within the semiclassical framework.
\end{abstract}

DOI: 10.1103/PhysRevD.104.105010

\section{INTRODUCTION}

Schwarzschild black-hole interiors contain geodesic borders separating them from spacelike singularities. Any information migrating across a geodesic border toward the singularity is irretrievably lost by causality. If Schwarzschild singularities absorb information, the corresponding evolution qualifies as paradoxical since it violates sacrosanct rules of information processing [1]. It is generally expected that spacetime fluctuations deform the Schwarzschild geometry near its geodesic border to yield a consistent quantum evolution. While the details of this dynamical regularization mechanism are unknown, they are important for global aspects of quantum information processing by black holes, such as the black-hole information paradox [2-4].

In this article, we show that Schwarzschild singularities border on asymptotically silent spacetime regions, that is regions inhibiting spatial quantum correlations irrespective of the initial field configuration. More importantly, they accommodate so-called Zeno borders, which mark a stack of hypersurfaces terminated by a geodesic border with the following property: The probability measure for populating quantum information within the stack decreases monotonously toward the singularity and vanishes at the geodesic border. As a consequence, quantum events cannot probe the geodesic border and quantum information cannot migrate

\footnotetext{
*1.eglseer@physik.uni-muenchen.de † stefan.hofmann@physik.uni-muenchen.de *marc.schneider@aei.mpg.de
}

Published by the American Physical Society under the terms of the Creative Commons Attribution 4.0 International license. Further distribution of this work must maintain attribution to the author(s) and the published article's title, journal citation, and DOI. Funded by SCOAP. across the geodesic border, irrespective of any quantum completion of gravity. In other words, Zeno borders render black-hole interiors leak-proof and represent, to the best or our knowledge, the first explicit quantum completion of a geodesically incomplete spacetime within the semiclassical framework [5]. Quantum field theoretic completeness conceptualizes the intrinsic consistency of quantum field theory in curved space-times in the sense that the theory itself is protected from a breakdown when reaching the geodesic border. Zeno regions incorporate this dynamical protection mechanism which guarantees the predictability of the theory itself and likewise passes the regularity on to physical observables.

To substantiate this probabilistic completion we proceed as follows: after stating the geometrical setup, we will present an intuitive argument based on scaling relations in the micropædia which introduces the idea of the Zeno region from the perspective of quantum measurements. The exact argument is then given in the macropædia followed by an interpretation based on observables showing that the probabilistic completion closes the geodesic border with respect to quantum field theory.

\section{GEOMETRICAL PRELIMINARIES}

Schwarzschild black holes are the warped geometries $\mathcal{B}:=P_{<} \times{ }_{t} S^{2}$, with $P_{<}$denoting the region $t<t_{\mathrm{g}}:=2 M$ in the $(t, r)$-half plane $\left(0, r_{\mathrm{g}}\right) \times \mathbb{R}^{+}$, where the projection $t: \mathcal{B} \rightarrow\left(0, r_{\mathrm{g}}\right)$ is the Schwarzschild time and the projection $r: \mathcal{B} \rightarrow \mathbb{R}^{+}$is the Schwarzschild radius function, and $S^{2}$ denotes the unit two-sphere. Note that in these conventions the Schwarzschild coordinate vector field $\partial_{t}$ is timelike, and $\partial_{r}$ is spacelike on $\mathcal{B}$. Compared to the Schwarzschild exterior spacetime, Schwarzschild time and radius function have interchanged their meaning. Taking this into account, the quadratic form associated with Schwarzschild black 
holes is $\mathrm{d} s^{2}=-s^{-1}(t) \mathrm{d} t^{2}+s(t) \mathrm{d} r^{2}+t^{2} w$, where $s(t):=$ $\left|1-r_{\mathrm{g}} / t\right|$ is the Schwarzschild function, and $w$ denotes the line element associated with the Euclidean metric on $S^{2}$, equipped with the usual spherical coordinates $(\vartheta, \varphi)$. $\mathcal{B}$ corresponds to a spatially homogeneous and anisotropic cosmological solution of general relativity. It can be foliated into Cauchy hypersurfaces $\left(0, r_{\mathrm{g}}\right) \times \Sigma$ along the temporal direction, where $\Sigma$ is the folio of spatial hypersurfaces $\Sigma_{t}$ labeled by Schwarzschild time. The metric field associated with the above quadratic form will be denoted by $g$, and its pull-back to $\Sigma$ by $g_{\Sigma}$.

In this geometry, the geodesic motion of a point particle that is initially equatorial relative to Schwarzschild spherical coordinates is bound to remain equatorial, $\vartheta=\pi / 2$. The so-called energy equation $E^{2}=(\mathrm{d} t / \mathrm{d} s)^{2}+V_{\text {eff }}$ holds, where $E:=s(t) \mathrm{d} r / \mathrm{d} s$ and $L:=t^{2} \mathrm{~d} \varphi / \mathrm{d} s$ are constants, which have an intuitive interpretation in the exterior as asymptotic energy per unit mass and angular momentum per unit mass, respectively. In fact, in the exterior, the definition of $L$ formally coincides with Kepler's second law. The effective potential is given by $V_{\text {eff }}:=-\left(1+L^{2} / t^{2}\right) s(t)$. Close to the endpoint at $t=0$, the effective potential is bounded from above, $V_{\text {eff }}=$ $-L^{2} r_{\mathrm{g}} / t^{3}$ plus less singular contributions. Thus the classical motion generated by $V_{\text {eff }}$ is incomplete at $t=0$. Potential incompleteness implies that $\mathcal{B}$ is geodesic incomplete [7], which in turn qualifies $\Sigma_{0}$ as a geodesic information sink. Geodesic incompleteness, however, does not imply quantum incompleteness (and vice versa).

\section{MICROPFEIA}

Let us first give an intuitive argument based on scaling relations showing that quantum information in $\mathcal{B}$ cannot migrate across $\Sigma_{0^{+}}$, before providing precise statements. In $\mathcal{B}$, scalar quantum fields $\Phi^{*}, \Phi$ charged under $U(1)$ evolve according to the Lagrange density $\mathcal{L}=\mathcal{L}_{0}+\mathcal{L}_{\text {int }}$, with the first term denoting the free theory $\mathcal{L}_{0}=\Phi^{*} \square \Phi$. The intuitive argument will be given in the absence of interactions, $\mathcal{L}_{\text {int }} \equiv 0$. Close to the spacelike singularity $\Sigma_{0}$ bordering on $\mathcal{B}, \quad \mathrm{d} s^{2} \cong-\left(t / r_{\mathrm{g}}\right) \mathrm{d} t^{2}+\left(r_{\mathrm{g}} / t\right) \mathrm{d} r^{2}+t^{2} w$, where $\cong$ means equality up to subleading contributions in each term as $\Sigma_{0}$ is approached [8]. In this asymptotic regime, $\square \cong\left(-r_{\mathrm{g}} / t\right)\left(\partial_{t}^{2}+(1 / t) \partial_{t}\right)+\left(t / r_{\mathrm{g}}\right) \partial_{r}^{2}+\left(1 / t^{2}\right) \partial_{\varangle}^{2}$. Here $\partial_{\varangle}^{2}$ denotes the usual angular part of the Laplace operator in $\mathbb{R}^{3}$ in Schwarzschild spherical coordinates.

The corresponding Green function $G$ is sourced by $\delta\left(t-t^{\prime}\right) \delta\left(\sigma-\sigma^{\prime}\right) / \sqrt{-\operatorname{det}(g)}$, with $\sigma$ and $\sigma^{\prime}$ denoting Schwarzschild spatial coordinates of events localized on $\Sigma_{t}$ and $\Sigma_{t^{\prime}}$, respectively, and satisfies

$$
\begin{aligned}
D(t) G\left(t, \sigma ; t^{\prime}, \sigma^{\prime}\right) & =\delta\left(t-t^{\prime}\right) \delta\left(\sigma-\sigma^{\prime}\right), \\
D(t) & :=-r_{\mathrm{g}} \partial_{t}\left(t \partial_{t}\right)+\left(t / r_{\mathrm{g}}\right) t^{2} \partial_{r}^{2}+\partial_{\varangle}^{2} .
\end{aligned}
$$

In order to estimate the asymptotic relevance of each term in the differential operator, consider $D(\varepsilon \tau)$ in the limit $\varepsilon \rightarrow 0^{+}$. The effective potential for free fields scales asymptotically as $1 / t^{2}$ and develops a repulsive barrier. It is well known from quantum mechanics that potentials of this type cannot be penetrated via tunneling processes.

Following the geometrical description of spacelike singularities by Belinskii, Khalatnikov, and Lifshitz [9], temporal variations dominate over spatial variations in the region bordering on $\Sigma_{0^{+}}$. Therefore, $D(\varepsilon \tau) \cong(1 / \varepsilon) \partial_{\tau}\left(\tau \partial_{\tau}\right)$. The time-dependent part of the source distribution scales like $\delta\left(\varepsilon \tau-t^{\prime}\right)$. This effectively allows to split the Green function $G=T\left(t, t^{\prime}\right) P\left(\sigma, \sigma^{\prime}\right)$ in the vicinity of $\Sigma_{0^{+}}$, with the asymptotic dynamics given by $\partial_{\tau}\left(\tau \partial_{\tau} T\right) \cong 0$. Here, all identifiers labeling the eigenvalue problem of the Laplace operator have been suppressed for ease of notation. We find the asymptotic solution $T\left(t, t^{\prime}\right) \cong C_{0}\left(t^{\prime}\right)+$ $C_{1}\left(t^{\prime}\right) \ln \left(t / r_{\mathrm{g}}\right)$, with $C_{0,1}<\infty$ depending on the initial values given on $\Sigma_{t^{\prime}}$.

In order to appreciate the rather mild divergence of the asymptotic solution $T$, we introduce a model for a physical detection with an emitter $F_{\text {em }}$ localized on $\Sigma_{t^{\prime}}, t^{\prime} \in\left(0, t_{*}\right)$ in the asymptotic domain, where $t_{*}$ denotes a fiducial time in this regime, and an absorber $F_{\mathrm{ab}}$ on $\Sigma_{\varepsilon \tau}$. A general measurement is given by a vertex density and involves the communication channel between source and the detector. For instance, consider $F_{\mathrm{ab}}=\delta(t-\varepsilon \tau) f_{\mathrm{ab}}(\sigma)$, with $f_{\mathrm{ab}}$ encoding the spatial extension of the detector on $\Sigma_{\varepsilon \tau}$. This blueprint effectively replaces part of $\Sigma_{0^{+}}$with a detector volume that can resolve arbitrary frequencies. Note that the asymptotic regime is controlled by the parameter $\varepsilon$ while $\tau$ represents a constant instant of time. The classical measurement process is described by the on-shell vertex density $\nu_{\mathrm{obs}}=\sqrt{-\operatorname{det}(g)} F_{\mathrm{ab}}^{*} \Xi_{\mathrm{os}}+$ c.c. with $\Xi_{\mathrm{os}}=G * F_{\mathrm{em}}$ denoting a convolution of $F_{\text {em }}$ with a bi-local kernel given by $G$ with respect to $x^{\prime}$ [10]. In the region bordering on $\Sigma_{0^{+}}$, as specified by the support properties of emitter and absorber, $\nu_{\text {obs }} \cong t^{2} \ln (t) \delta(t-\varepsilon \tau) \sin (\vartheta) f_{\mathrm{ab}} F_{\mathrm{em}}$, where $F_{\text {em }}$ contains the exclusive information on the emission process and depends only on source parameters. In particular, $F_{\mathrm{em}}$ is finite in accordance with the homogeneous interior $\mathcal{B}$. For fixed $t^{\prime}$ and $\varepsilon \rightarrow 0$, the measurement of the emitter's influence on the detector gives a vanishing response, see figure $1, \nu_{\mathrm{obs}} \cong 0$, in the distributional sense. This implies that no information carried by local bookkeeping devices reach $\Sigma_{0^{+}}$.

It is possible to be more specific about the emitter and absorber. As an example, the energy momentum tensor for the complex scalar field scales like $\mathcal{T}=\mathrm{d} \Phi \otimes \mathrm{d} \Phi^{*} \propto$ $1 /(\varepsilon \tau)^{2}$ on $\Sigma_{\varepsilon \tau}$ and develops a singularity toward $\Sigma_{0^{+}}$. Our detector model is given by $F_{\mathrm{ab}}=\delta(t-\varepsilon \tau) f_{\mathrm{ab}}(\sigma)$, with $f_{\mathrm{ab}}$ encoding the spatial extension of the detector on $\Sigma_{\varepsilon \tau}$ or a more physical absorber of an ideal gas $\tilde{F}_{\mathrm{ab}}=M(\varepsilon \tau) U \otimes U$, where $U \cong \sqrt{\varepsilon \tau / r_{\mathrm{g}}} \mathrm{d} t$ is the normal, and $M(t)$ denotes the spatial volume integral over an 


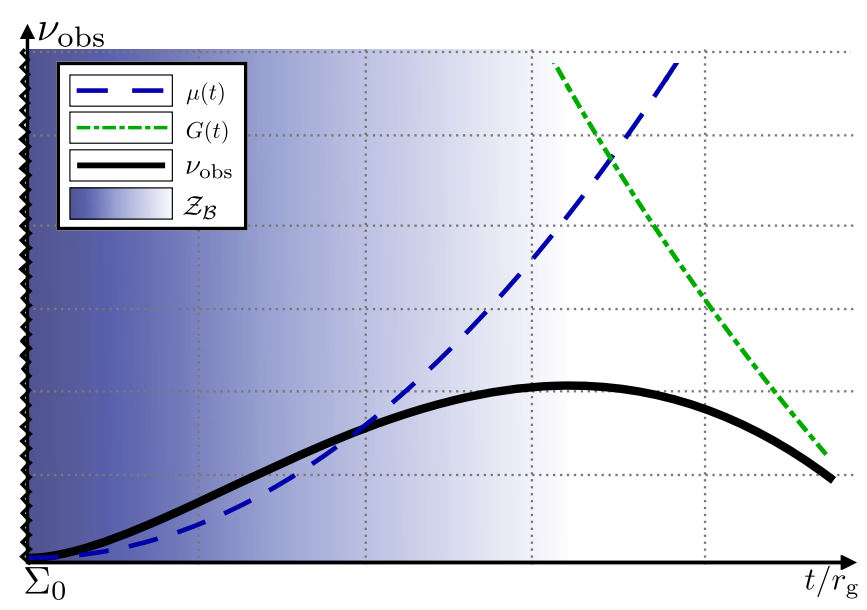

FIG. 1. Vertex density $\nu_{\mathrm{obs}}$ for measurement processes inside Schwarzschild black holes. The communication between emitter and absorber, described by the asymptotic Green function $G$, diverges toward the geodesic border $\Sigma_{0}$, but the integration measure $\mu$ goes sufficiently fast to zero to render $\nu_{\text {obs }}$ finite at all times. Furthermore, within the Zeno region (shaded), see Fig. 2 and the macropadia for details, $\nu_{\mathrm{obs}}$ is monotonously decreasing and vanishes toward $\Sigma_{0}$.

energy density. From a phenomenological point of view, $F_{\text {ab }}$ is required to have nontrivial support toward $\Sigma_{0^{+}}$. Then, $\tilde{\nu}_{\text {obs }} \cong 0$, as well, which only confirms that the asymptotic description of the tree-level measurement process is independent of the tensor providing the principal communication channel.

Before closing the micropæ dia, let us briefly discuss the asymptotic diagnostics of Noether charges. Let $\Phi: \mathcal{B} \rightarrow \mathbb{C}$ carry a $U(1)$ charge. The four-dimensional $U(1)$ current density is $j=i\left(\Phi^{*} P \Phi-\right.$ c.c. $)$, where $P$ denotes the fourmomentum operator. Projecting the current density onto $U$ from before, we find the following scaling relation for the charge density $\rho$ localized on $\Sigma_{\varepsilon \tau}: \rho(\varepsilon \tau) \cong \rho\left(t_{*}\right)\left(t_{*} / \varepsilon \tau\right)^{3 / 2}$, which formally diverges as $\Sigma_{0^{+}}$is approached. Physical measurements of the charge $Q(\varepsilon \tau)$, however, yield a finite result. In fact $Q(\varepsilon \tau)=Q\left(t_{*}\right)$. Black holes cannot be discharged through the geodesic singularity bordering on $\Sigma_{0^{+}}$. Any active information sink would necessarily lead to charge depletion. Note that this discussion of asymptotic charge conservation is fully based on local physics inside black holes, and no reference to the usual global characterization in the exterior is made.

\section{MACROP FDIA}

A more rigorous argument is based on the Schrödinger representation of local quantum physics which we summarize for convenience below. Let $(a, b) \times \Sigma$ be a Cauchy foliation of a globally hyperbolic spacetime $(\mathcal{M}, g)$ with a geodesic border $\Sigma_{a}$ in the folio $\Sigma$ of hypersurfaces. For $t \in(a, b)$, we denote by $\mathcal{C}\left(\Sigma_{t}\right)$ the space of instantaneous field configurations $\phi: \Sigma_{t} \rightarrow \mathbb{C}$. Consider the $\mathbb{C}$-vector space of measurable wave functionals $\Psi_{t}: \mathcal{C}\left(\Sigma_{t}\right) \rightarrow \mathbb{C}$ whose modulus is square integrable with respect to the formal functional measure $\mathcal{D} \phi$. Let $L^{2}\left(\mathcal{C}\left(\Sigma_{t}\right), \mathcal{D} \phi\right)$ be the quotient of this space by the subspace of wave functionals vanishing $\mathcal{D} \phi$-almost everywhere in $\mathcal{C}\left(\Sigma_{t}\right)$. We introduce the usual norm on $L^{2}\left(\mathcal{C}\left(\Sigma_{t}\right), \mathcal{D} \phi\right)$. If $\mathcal{U}$ is a formally measurable subset of $\mathcal{C}\left(\Sigma_{t}\right)$ and $\mathcal{X}_{\mathcal{U}}$ the corresponding indicator functional, then $\left\|\mathcal{X}_{\mathcal{U}} \Psi_{t}\right\|^{2}$ is the probability for the field configuration on $\Sigma_{t}$ to be given by some $\phi \in \mathcal{U}$. This interpretation requires normalizable wave functionals. The configuration field operator $\Phi[f]$ is just the multiplication operator with $\phi[f]$, where $f$ denotes some smooth test function of compact support on $\Sigma_{t}$ such that $\left\langle\Psi_{t}|\Phi[f]| \Psi_{t}\right\rangle=\left\|\sqrt{\phi[f]} \Psi_{t}\right\|^{2}$ is bounded. The conjugated momentum field operator $\Pi[f]$ is the functional derivative $-\mathrm{i}\left(\operatorname{det}\left(g_{\Sigma_{t}}\right)\right)^{-1 / 2} \delta / \delta \phi$ in the direction of $f$. Heisenberg's fundamental uncertainty relation is a consequence of $\left[\Phi\left[f_{1}\right], \Pi\left[f_{2}\right]\right]=\mathrm{i}\left(f_{1}, f_{2}\right)$, where $(\cdot, \cdot)$ denotes the scalar product of smearing functions on $\Sigma_{t}$.

Consider the evolution semigroup given by the continuous map $(a, b) \rightarrow L^{2}\left(\mathcal{C}\left(\Sigma_{t}\right), \mathcal{D} \phi\right)$, defined by $t \rightarrow$ $\mathcal{E}\left(t, t_{0}\right)\left|\Psi_{t_{0}}\right\rangle$ for an initial time $t_{0} \in(a, b)$. A probabilistic interpretation is only possible for $\left\|\mathcal{E}\left(t, t_{0}\right)\right\| \leq 1$, that is for contractive evolution semigroups. While this includes unitarity as a special case for Minkowski spacetime, its generator density $\mathcal{H}$ is only required to be accretive, $\operatorname{Re}\left(\left\langle\Psi_{t}|\mathcal{H}| \Psi_{t}\right\rangle\right) \geq 0$, instead of being self-adjoint. On generic spacetimes, the pair (contractive, accretive) supersedes (unitary, self-adjoint) as a requirement on evolution semigroups in order to allow for a probabilistic interpretation. This has important ramifications for physical spacetimes as support for quantum events:

Let $\left(L^{2}\left(\mathcal{C}\left(\Sigma_{t_{0}}\right)\right), \mathcal{H}\right)$ be the initial Schrödinger representation of a quantum field theory in a globally hyperbolic spacetime $(M, g)$ with the Cauchy foliation $(a, b) \times \Sigma$ containing a geodesic boundary $\Sigma_{a}$ in the folio $\Sigma$ of hypersurfaces. A Zeno region $\mathcal{Z}_{\mathcal{M}}$ in $\mathcal{M}$ is a stack of Cauchy hypersurfaces in $\Sigma$ with the following property: The evolution semigroup generated by $\mathcal{H}$ is strictly contractive in $\mathcal{Z}_{\mathcal{M}}$ and $\left\|\mathcal{E}\left(c, t_{0}\right)\right\| \rightarrow 0$ as $c \rightarrow a$.

Evidently, $\mathcal{Z}_{\mathcal{M}}$ is bounded by some Cauchy hypersurface $\Sigma_{z}$, called the Zeno border, and the geodesic border $\Sigma_{a}$. Let us justify the introduction of Zeno regions: If a spacelike singularity borders on a Zeno region, then the singularity cannot be probed by quantum fields and the quantum evolution in its immediate vicinity allows for a probabilistic interpretation. This distinguishes the physical spacetime as the principal support of quantum events from the geometrical spacetime model. Therefore Zeno regions imply a probabilistic or quantum completion of a geodesic incomplete spacetime within the semiclassical framework.

Let us now turn back to Schwarzschild black-hole interiors $\mathcal{B}$, Cauchy-foliated as $\left(0, r_{\mathrm{g}}\right) \times \Sigma$ with respect to the Schwarzschild time function. Consider the space $\mathcal{C}\left(\Sigma_{t_{0}}\right)$ of instantaneous field configuration $\phi, \phi^{*}: \Sigma_{t_{0}} \rightarrow \mathbb{C}$ 
and its Schrödinger representation $\left(L^{2}\left(\mathcal{C}\left(\Sigma_{t_{0}}\right)\right), \mathcal{H}\right)$ in $\mathcal{B}$ with Hamilton density $\mathcal{H}=\mathcal{G}\left(\Pi^{*}, \Pi\right)+\mathcal{V}\left(\Phi^{*}, \Phi\right)$, where $\mathcal{G}\left(\Pi^{*}, \Pi\right):=\sqrt{-g_{t t}} \Pi^{*} \Pi / \operatorname{det}\left(g_{\Sigma_{t}}\right), \quad$ and $\quad \Pi:=-\mathrm{i} \delta / \delta \Phi$ denotes the momentum field conjugated to $\Phi$ [11]. The effective potential density $\mathcal{V}:=\sqrt{-g_{t t}}\left(|\operatorname{grad} \Phi|^{2}+m^{2}|\Phi|^{2}\right)$ is a pure multiplication operator, with $m$ being the mass of the scalar field.

For the ground-state wave functional we make the following ansatz

$$
\Psi_{t}\left[\phi^{*}, \phi\right]=\mathcal{N}_{t} \exp \left(-\left[V^{*}\right] \mathcal{K}_{t}[V]\right),
$$

which will be justified a posteriori. Here, $V=\left(\phi, \phi^{*}\right)^{\mathrm{T}}$, and

$$
\left[V^{*}\right] \mathcal{K}_{t}[V]:=\frac{1}{2} \int_{\Sigma_{t}} \mathrm{~d} \mu_{x, y} V^{*}(x) K_{t}(x, y) V(y)
$$

is a quadratic functional with a bilocal representation given by the matrix $K$. In the absence of interactions, $K$ is diagonal and only its trace enters in (3). Close to the Schwarzschild singularity $\Sigma_{0}$ the quantum evolution trivializes [12], that is $\mathcal{H} \approx \mathcal{G}\left(\Pi^{*}, \Pi\right)$. As a consequence, the trace of the bilocal kernel matrix $K$ becomes a contact term on $\Sigma_{t} \in \Sigma$ near the geodesic border, $\operatorname{tr}\left(K_{\varepsilon \tau}\right)(x, y) \cong$ $k(\varepsilon \tau) \delta^{(3)}(x, y)$ with [13]

$$
\begin{aligned}
& \operatorname{Im}(k(\varepsilon \tau)) \cong-\frac{2}{(\varepsilon \tau)^{3}} \frac{1}{|\ln (\varepsilon \tau)|}, \\
& \operatorname{Re}(k(\varepsilon \tau)) \cong|\operatorname{Im}(C)| \frac{|\operatorname{Im}(k(\varepsilon \tau))|}{|\ln (\varepsilon \tau)|},
\end{aligned}
$$

where $\cong$ refers to the limit $\varepsilon \rightarrow 0$. Our result is reminiscent of the analysis of Kasner spacetimes by Belinskii, Khalatnikov and Lifshitz: In the vicinity of spacelike singularities, but still in the domain of general relativity, the spatial variations of local quantities are insignificant compared to temporal gradients. This is also similar to asymptotic silence [14] which describes how the particle horizon shrinks in homogeneous but anisotropic spacetimes bordering on a spacelike singularity. All spatial correlations vanish eventually.

The asymptotic kernel (4) implies that the immediate vicinity of the geodesic border is a Zeno region $\mathcal{Z}_{\mathcal{B}}$ since

$$
\lim _{\varepsilon \rightarrow 0} \Psi_{\varepsilon \tau}\left[\phi^{*}, \phi\right]=\lim _{\varepsilon \rightarrow 0}|\ln (\varepsilon \tau)|^{-\Lambda v\left(\Sigma_{\varepsilon \tau}\right)}=0,
$$

where $\Lambda$ denotes a short-distance cutoff and $v\left(\Sigma_{\varepsilon \tau}\right)$ a volume regularization. Note that any hypersurface contained in $\mathcal{Z}_{\mathcal{B}}$ has an infinite volume, (5) is robust against the detailed regularization prescription. The Zeno region $\mathcal{Z}_{\mathcal{B}}$ secludes the Schwarzschild black-hole's interior not granting any sojourn to quantum probes on the singular hypersurface bordering on $\mathcal{B}$. Thence the physical space-time is closed for quantum probes despite being geodesically incomplete in the mathematical notion. In other words, it

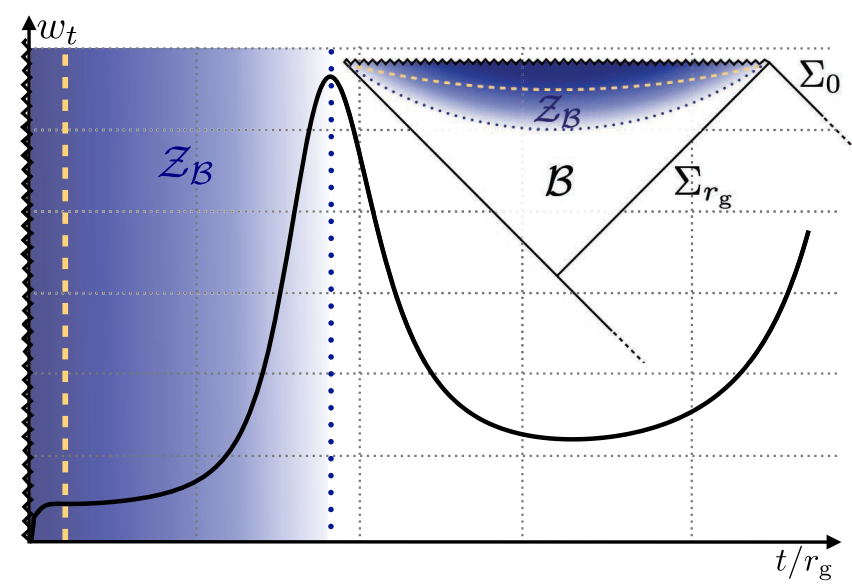

FIG. 2. Probability density $w_{t}^{\ell}=\left|\Psi_{t}^{\ell}\left[\phi^{*}, \phi\right]\right|^{2}$ for the distribution of a field configuration $\phi$ for a fixed $\ell$ in the folium $\Sigma$ of $\mathcal{B}$. Wavy lines mark the geodesic border $\Sigma_{0}$, dashed lines represent the onset of asymptotic silence, and the shaded region between $\Sigma_{0}$ and the dotted line is the Zeno region $\mathcal{Z}_{\mathcal{B}}$. The upper right corner displays the corresponding Penrose diagram.

is the portion of $\mathcal{B}$ that can be probed via the support of quantum events, see Fig. 2.

Intuitively $\mathcal{Z}_{\mathcal{B}}$ marks the stack of hypersurfaces which enforces the probabilistic completion such that the geodesic border is closed in the quantum theoretical sector. Quantum field theory, hence, introduces a new scale $\tau_{\mathcal{Z}}$ in the black hole interior. To classify $\tau_{\mathcal{Z}}$ further, we compare it to the scales coming from classical gravity, i.e., $r_{\mathrm{g}}$, and from quantum gravity, i.e., the Planck time $t_{\mathrm{Pl}}$.

Following the definition of $\mathcal{Z}_{\mathcal{B}}$, the exact location is given by an interplay between the background geometry and the probing quanta. The relevant quantity to estimate $\tau_{\mathcal{Z}}$ is the position of the maximum in the probability density $w_{\varepsilon \tau}=\left|\Psi_{\varepsilon \tau}\left[\phi^{*}, \phi\right]\right|^{2}$ which is closest to $\Sigma_{0}$. Since the asymptotic solution is confined to a small region, we need to extend it by including less singular terms of order $\mathcal{O}(1 / \varepsilon)$ such that $\Psi_{\varepsilon \tau}$ covers the evolution of test fields in a larger portion of $\mathcal{B}$ and a possible maximum becomes visible. In this range, we find oscillatory solutions to (1) given by Bessel functions. This allows to estimate the position of $\tau_{\mathcal{Z}}$ at which the maximum occurs which also determines the beginning of the Zeno region. To give a proof of concept, we perform a mode-sum decomposition in $l \in \mathbb{N}_{0}$, where we split off the time-dependent contribution of the kernel (note, for the full kernel the sum over all $l$ has to be performed)

$k_{\ell}(\varepsilon \tau)=\frac{-i}{\varepsilon^{3} \tau^{2}} \partial_{\tau} \ln \left(c_{1} \mathrm{~J}_{0}\left(\sqrt{\ell^{2} \varepsilon \tau}\right)+c_{2} \mathrm{Y}_{0}\left(\sqrt{\ell^{2} \varepsilon \tau}\right)\right)$.

Here, $\ell^{2}=4 l(l+1), c_{i} \in \mathbb{C}$ constants of integration, and $\mathrm{J}_{0}$ and $\mathrm{Y}_{0}$ are the Bessel function of the first and second kind. The location of $\Sigma_{\mathcal{Z}}^{\ell}$ can be expressed as a threeparameter family of hypersurfaces determined by $\ell^{2}$ as well 
as the initial conditions represented by $c_{1}$ and $c_{2}$ of the quantum probes. By performing a series expansion for small arguments $\ell^{2} \varepsilon \tau \ll 1$, we can approximate the position of the maximum in $w_{\tau}^{\ell}$ for $\ell^{2}$-values that allow for a suitable covering in the $\tau$-direction within this approximation. Thus, we find for a given $\ell$

$$
\tau_{\mathcal{Z}}^{\ell} \approx \frac{r_{\mathrm{g}}}{\left|\mathcal{C}\left(c_{1}, c_{2}\right)\right|^{2} \ell^{2}},
$$

where $\mathcal{C}\left(c_{1}, c_{2}\right)$ is a constant depending on the initial conditions. Apparently, increasing the angular momentum $\ell$ results in shifting the Zeno border closer to the geodesic border while for the case $\ell=0$ we find the logarithmic (non-oscillating) solution in (5) where, the Zeno border lies at infinity, i.e., it covers all of the interior. Although the exact position of $\mathcal{Z}_{\mathcal{B}}$ depends on the specifics of the solution, we can compare $\tau_{\mathcal{Z}}^{\ell}$ to the high-energy scale provided by the Planck energy. To this aim, we consider Christensen's general form of a renormalized stress-energy tensor [15] with Synge's world function given in [16] and look at its trace $\langle T(x)\rangle_{\text {ren }}=\frac{1}{8 \pi^{2}} \sqrt{-\operatorname{det}(g)}\left(\frac{1}{360} K+C\right)+$ $\mathcal{O}\left(1 / m^{2}\right) \approx r_{\mathrm{g}}^{2} /\left(224 \pi^{2} \tau^{4}\right)$ where $K=12 r_{\mathrm{g}}^{2} / \tau^{6}$, the Kretschmann scalar and $C=\frac{1}{432} r_{\mathrm{g}}^{2} / \tau^{6}$ other contractions of the Riemann tensor. The trace is usually the most divergent contribution since it includes the inverse metric. By comparing it with the Planck scale $t_{\mathrm{Pl}}$, we are able to determine $\tau_{\star}$ where presumably quantum gravity effects become important. Therefore, we demand $\left\langle T\left(\tau_{\star}\right)\right\rangle_{\mathrm{ren}}=t_{\mathrm{Pl}}^{-2}$. Hence, we locate the high-energy scale at $\tau_{\star} \approx \frac{1}{7} \sqrt{r_{\mathrm{g}} t_{\mathrm{Pl}}}$. For stellar-mass Schwarzschild black holes, the Zeno border will then be located $\tau_{\star}<\tau_{\mathcal{Z}}^{\ell} \leq r_{\mathrm{g}}$ for all initial conditions coming from sub-Planckian energy densities.

Next we consider observables in $\mathcal{Z}_{\mathcal{B}}$ and introduce an auxiliary source functional $\mathcal{J}: \mathcal{C}\left(\Sigma_{t}\right) \rightarrow \mathbb{C}$, describing the absorption and emission of an instantaneous field configuration by the associated local current density $J: \Sigma_{t} \rightarrow \mathbb{C}$. Let $\Psi_{t}^{J}[\phi]:=\left\langle\phi\left|\exp \left(\mathcal{J}_{t}\right)[\Phi]\right| \Psi_{t}\right\rangle$ which allows to replace compositions of the configuration operator $\Phi$ by the corresponding succession of functional derivatives with respect to the current. In the presence of the auxiliary source,

$$
\begin{aligned}
& \left\langle\Psi_{t}^{J} \|\left.\Phi[f]\right|^{2} \mid \Psi_{t}^{J}\right\rangle \\
& \quad=[f] \delta_{J}^{2} \exp \left\{\frac{1}{4} \frac{1}{\sqrt{\operatorname{det}\left(g_{\Sigma}\right)}}[J]\left[\operatorname{Re}\left(\mathcal{K}_{t}\right)\right]^{-1}[J]\right\}\left\|\Psi_{t}^{J}[\phi]\right\|^{2}
\end{aligned}
$$

with $[f] \delta_{J}^{2}$ denoting the second functional derivative with respect to $J$, smeared with an appropriate field configuration $f$. In the absence of an auxiliary source, the ground-state expectation (8) is real and semipositive definite. It is monotonously decreasing in $\mathcal{Z}_{\mathcal{B}}$ toward $\Sigma_{0}$ and vanishes on $\Sigma_{0}$. Similarly,

$$
\begin{aligned}
& \left\langle\Psi_{t}^{J} \|\left.\Pi\right|^{2}[f] \mid \Psi_{t}^{J}\right\rangle \\
& \quad=\left.[f] \mathcal{K}_{t}[f]\left\|\Psi_{t}^{J}[\phi]\right\|\right|^{2}-\operatorname{det}\left(g_{\Sigma}\right) k^{2}(t)\left\langle\left.\Psi_{t}^{J}|| \Phi[f]\right|^{2} \mid \Psi_{t}^{J}\right\rangle_{\Sigma_{t}} .
\end{aligned}
$$

Both, the real and imaginary part of (9) are monotonously decreasing in $\mathcal{Z}_{\mathcal{B}}$ toward $\Sigma_{0}$ and vanish on $\Sigma_{0}$ when the auxiliary current is switched off.

Within the Zeno region, the ground-state expectation values (8) and (9) are controlled by the contractive representation of the evolution semigroup. As a result $\left\langle\Psi_{t}^{J}|\mathcal{H}[f]| \Psi_{t}^{J}\right\rangle$ does receive decreasing probabilistic support within $\mathcal{Z}_{\mathcal{B}}$ and has vanishing probabilistic support on $\Sigma_{0}$. The Zeno region completes $\mathcal{B}$ since information cannot leak through the geodesic information sink $\Sigma_{0}$. These results remain true if (self-) interactions are included [12] which can be understood at the qualitative level from the approximate operator identity $\mathcal{H} \cong \mathcal{G}\left(\Pi^{*}, \Pi\right)$.

\section{DISCUSSION}

The mathematical spacetime model of Schwarzschild black-hole interiors is geodesic incomplete. This result is often used to argue for the breakdown of general relativity as predicted by general relativity. In this letter we have shown that interiors of Schwarzschild black holes contain so-called Zeno regions, i.e., stacks of hypersurfaces in the vicinity of the geodesic spacetime singularity, on which observables enjoy a vanishing probabilistic support toward the geodesic spacetime border. In this sense Zeno regions represent a probabilistic completion of Schwarzschild black holes within the usual semiclassical framework. Moreover, geodesic incompleteness of Schwarzschild black holes qualifies the mathematical spacetime model, but certainly not the physical spacetime with a Zeno region in the immediate vicinity of the geodesic singularity. This statement is justified since Zeno regions allow for a consistent evolution of quantum fields but are beyond the scope of classical point particle mechanics. It is interesting to speculate whether Zeno regions are terminated by Hawking's hidden surface to which the principle of ignorance applies [17], but this we leave for further investigations.

\section{ACKNOWLEDGMENTS}

Thanks to Maximilian Kögler, Robert Myers, Florian Niedermann, and Kerstin Paech for delightful discussions and their thoughts on the topic. We appreciate financial support from the Alexander von Humboldt-Stiftung. 
[1] G. T. Horowitz and J. Maldacena, J. High Energy Phys. 02 (2004) 008.

[2] S. D. Mathur, Classical Quantum Gravity 26, 224001 (2009).

[3] S. B. Giddings, Phys. Rev. D 85, 124063 (2012).

[4] D. Marolf, Rep. Prog. Phys. 80, 092001 (2017).

[5] A probabilistic completion as presented here, has been formerly described in quantum mechanics, e.g., for timelike singularities [6].

[6] G. T. Horowitz and D. Marolf, Phys. Rev. D 52, 5670 (1995).

[7] S. Hawking and R. Penrose, Proc. R. Soc. A 314, 529 (1970).

[8] For $\vartheta$ small, the quadratic form can be transformed into type-D Kasner line-element with exponents $\left(p_{1}, p_{2}, p_{3}\right)=$ $(2 / 3,2 / 3,-1 / 3)$, corresponding to a spatially anisotropic cosmology.
[9] V. A. Belinskii, I. M. Khalatnikov, and E. M. Lifshitz, Adv. Phys. 19, 525 (1970).

[10] B. DeWitt, The Global Approach to Quantum Field Theory (Oxford University Press, New York, 2003), Vol. 114.

[11] C. Kiefer, Phys. Rev. D 45, 2044 (1992).

[12] S. Hofmann and M. Schneider, Phys. Rev. D 95, 065033 (2017).

[13] S. Hofmann and M. Schneider, Phys. Rev. D 91, 125028 (2015).

[14] L. Andersson, H. van Elst, W. C. Lim, and C. Uggla, Phys. Rev. Lett. 94, 051101 (2005).

[15] S. M. Christensen, Phys. Rev. D 14, 2490 (1976).

[16] A. Levi and A. Ori, Phys. Rev. D 91, 104028 (2015).

[17] S. W. Hawking, Phys. Rev. D 14, 2460 (1976). 